\title{
Modulus design multiwavelength polarization microscope for transmission Mueller matrix imaging
}

\author{
Jialing Zhou \\ Honghui He \\ Zhenhua Chen \\ Ye Wang \\ Hui Ma
}




\title{
Modulus design multiwavelength polarization microscope for transmission Mueller matrix imaging
}

\author{
Jialing Zhou, ${ }^{\mathrm{a}, \mathrm{b}}$ Honghui He, ${ }^{\mathrm{a}}$ Zhenhua Chen, ${ }^{\mathrm{c}}$ Ye Wang, ${ }^{\mathrm{a}, \mathrm{d}}$ and Hui Ma ${ }^{\mathrm{a}, \mathrm{c}, \mathrm{d}, \star}$ \\ ${ }^{a}$ Tsinghua University, Shenzhen Key Laboratory for Minimal Invasive Medical Technologies, Institute of Optical Imaging and Sensing, \\ Graduate School at Shenzhen, Shenzhen, China \\ ${ }^{\mathrm{b}}$ Tsinghua University, Department of Biomedical Engineering, Beijing, China \\ 'Tsinghua-Berkeley Shenzhen Institute, Shenzhen, China \\ ${ }^{\mathrm{d}}$ Tsinghua University, Department of Physics, Beijing, China
}

\begin{abstract}
We have developed a polarization microscope based on a commercial transmission microscope. We replace the halogen light source by a collimated LED light source module of six different colors. We use achromatic polarized optical elements that can cover the six different wavelength ranges in the polarization state generator (PSG) and polarization state analyzer (PSA) modules. The dual-rotating wave plate method is used to measure the Mueller matrix of samples, which requires the simultaneous rotation of the two quarter-wave plates in both PSG and PSA at certain angular steps. A scientific CCD detector is used as the image receiving module. A LabView-based software is developed to control the rotation angels of the wave plates and the exposure time of the detector to allow the system to run fully automatically in preprogrammed schedules. Standard samples, such as air, polarizers, and quarter-wave plates, are used to calibrate the intrinsic Mueller matrix of optical components, such as the objectives, using the eigenvalue calibration method. Errors due to the images walk-off in the PSA are studied. Errors in the Mueller matrices are below 0.01 using air and polarizer as standard samples. Data analysis based on Mueller matrix transformation and Mueller matrix polarization decomposition is used to demonstrate the potential application of this microscope in pathological diagnosis. @ The Authors. Published by SPIE under a Creative Commons Attribution 3.0 Unported License. Distribution or reproduction of this work in whole or in part requires full attribution of the original publication, including its DOI. [DOI: 10.1117/1.JBO.23.1.016007]
\end{abstract}

Keywords: modulus design; polarization microscope; Mueller matrix; multiwavelength.

Paper 170619RR received Oct. 1, 2017; accepted for publication Dec. 14, 2017; published online Jan. 8, 2018.

\section{Introduction}

Polarization imaging techniques are capable of probing the microstructural and optical properties of samples. ${ }^{1,2}$ They have been applied in different fields, including material characterization, ${ }^{3,4}$ medical diagnosis, ${ }^{5}$ remote sensing, ${ }^{6}$ target detection, ${ }^{7}$ and so on. The observation of microstructure makes it necessary to combine polarization imaging techniques with microscope systems. Oldenbourg ${ }^{8}$ established a polarization microscope by adding liquid crystal polarization modulator to a commercial transmission microscope. Other attempts for polarization microscopes have also been reported. ${ }^{9-11}$ These microscopes use monochromatic light sources and qualitatively analyze the birefringence properties of the samples. For complicated samples with strong depolarization and complex anisotropy like various biological tissues and cells, birefringence alone is not enough to describe different polarization characteristics. Mueller matrix measurements are more appropriate for polarization characterization of biological tissues because it describes all of the polarization properties, such as diattenuation, retardance, and depolarization. ${ }^{12-17}$ The hardware of Mueller matrix measurement is mostly similar to the corresponding nonpolarized measurements except for the polarization modulation optics. Therefore, a normal transmission or reflection mode microscope can be converted to a Mueller matrix microscope by adding a polarization-state generator (PSG) and a polarization-state analyzer (PSA) to the existing optical path. Different wavelengths

*Address all correspondence to: Hui Ma, E-mail: mahui@tsinghua.edu.cn of light behave differently in scattering, absorption, and propagation processes of the same biological tissue. Therefore, the feature parameters used to describe the sample, such as retardance $\delta,{ }^{18}$ that is extracted from different wavelength Mueller matrices of the same biological tissue are also different. Compared with a monochromatic Mueller matrix microscope, polarization measurements with different wavelengths will bring up extra information on the microstructure of samples. For different biological samples or different feature parameters, we can take advantage of multiple wavelengths to choose an optimal wavelength to measure the Mueller matrix. Previously, we reported a monochromatic Mueller matrix microscope by adding PSG and PSA to a commercial transmission-light microscope $^{16}$ and demonstrated preliminary applications in detecting human cancerous tissues with fibrosis. ${ }^{2}$ In this paper, we report a multiwavelength version of the microscope and include detailed descriptions on how the microscope is designed, constructed, calibrated, and tested. The Mueller microscope is also based on an ordinary commercial optical microscope. We use a multiwavelength collimated LED light source module to substitute the ordinary halogen light source, add a set of polarizing and analyzing modules, and use a CCD detector as the image receiving module. The dual-rotating quarter-wave plates Mueller matrix measurement method is adopted to measure the Mueller matrix of samples by simultaneously rotating two quarter-wave plates at certain steps. ${ }^{19}$ For higher accuracy, optimizations and calibration processes are adopted to reduce the errors in the polarization measurements. With the advantages of simple structure, rapid measurement, 
and high precision, the modulus design polarization microscope for transmission Mueller matrix imaging shows promising prospects.

\section{Theory of Operations and Selection Criteria of Components}

\subsection{Theory of Operations}

After comparing the division of focal plane polarimeter ${ }^{20,21}$ and rotating both polarizers and quarter-wave plates Mueller matrix measurement, ${ }^{22}$ we selected the dual-rotating quarter-wave plates Mueller matrix measurement method for the polarization microscope. In this measurement, there are only two quarterwave plates synchronously rotating at a set of angular increments that introduce less error sources and are easy to be reconstructed and controlled by the computer. The main principle of the dual-rotating quarter-wave plates Mueller matrix measurement method is shown below.

Figure 1 shows that the state-of-polarization (SoP) of detecting light $S_{\text {out }}$ can be expressed with the Mueller matrix of components and the light intensity value is the first component of $S_{\text {out }}$; therefore, the light intensity $I_{\text {out }}$ can be expressed as the following equation:

$$
\begin{aligned}
I_{\mathrm{out}} & =\left(\begin{array}{llll}
1 & 0 & 0 & 0
\end{array}\right) S_{\mathrm{out}} \\
& =c\left(\begin{array}{llll}
1 & 0 & 0 & 0
\end{array}\right) M_{P 2} M_{R 2} M_{\mathrm{sample}} M_{R 1} M_{P 1} S_{\mathrm{in}}
\end{aligned}
$$

where $S_{\text {in }}$ and $S_{\text {out }}$ represent the Stokes vector of the incident nature light and the detecting light, respectively, $\mathrm{M}_{P 1}$ and

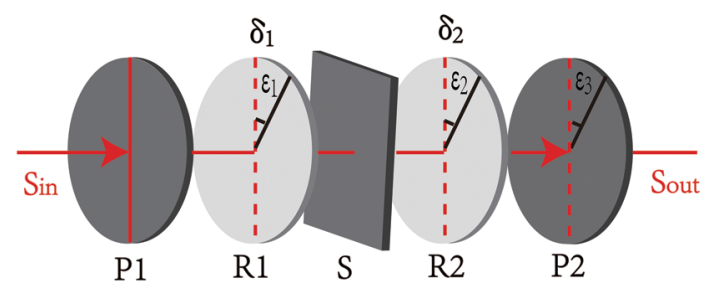

Fig. $1 S_{\text {in }}, S_{\text {out }}$ : the Stokes vectors of incident light and detecting light; $P 1, P 2$ : fixed polarizers; $R 1, R 2$ : rotating retarders; S: sample; $\varepsilon_{1}, \varepsilon_{2}, \varepsilon_{3}$ : the azimuthal alignment errors of $R 1, R 2, P 2$ relative to the transmission axis of $P 1 ; \delta_{1}, \delta_{2}$ : retardance errors.

$M_{R 1}$ represent the Mueller matrices of linear polarizer $P 1$ and quarter-wave plate $R 1$ in the PSG module, respectively, $M_{P 2}$ and $M_{R 2}$ represent the Mueller matrices of linear polarizer $P 2$ and quarter-wave plate R2 in the PSA module, respectively, $M_{\text {sample }}$ represents the Mueller matrix of the sample, and $c$ represents the detector sensitivity.

Let $P=M_{R 1} M_{P 1} S_{\text {in }}$, which represents the Stokes vector of light leaving the PSG module and $p_{j}$ represents the elements in the Stokes vector. Let $A=\left(\begin{array}{cccc}1 & 0 & 0 & 0\end{array}\right) M_{P 2} M_{R 2}$, which represents the first row of the Mueller matrix of PSA module, and $a_{i}$ represent the elements in the Stokes vector. Then, we can use $u_{i j}$ to represent the elements in the vector product of $P$ and $A$ and obtain the following expression:

$$
I_{\mathrm{out}}=\mathrm{c} A M_{\mathrm{sample}} P=\mathrm{c} \sum_{i, j=1}^{4} a_{i} p_{j} m_{i j}=\mathrm{c} \sum_{i, j=1}^{4} u_{i j} m_{i j}
$$

$$
u=1 / 4\left(\begin{array}{cccc}
1 & \cos ^{2} 2 \theta_{1} & \sin 2 \theta_{1} \cos 2 \theta_{1} & \sin 2 \theta_{1} \\
\cos ^{2} 2 \theta_{2} & \cos ^{2} 2 \theta_{1} \cos ^{2} 2 \theta_{2} & \sin 2 \theta_{1} \cos 2 \theta_{1} \cos ^{2} 2 \theta_{2} & \sin 2 \theta_{1} \cos ^{2} 2 \theta_{2} \\
\sin 2 \theta_{2} \cos 2 \theta_{2} & \cos ^{2} 2 \theta_{1} \sin 2 \theta_{2} \cos 2 \theta_{2} & \sin 2 \theta_{1} \sin 2 \theta_{2} \cos 2 \theta_{1} \cos 2 \theta_{2} & \sin 2 \theta_{1} \sin 2 \theta_{2} \cos 2 \theta_{2} \\
-\sin 2 \theta_{2} & -\cos ^{2} 2 \theta_{1} \sin 2 \theta_{2} & -\sin 2 \theta_{1} \sin 2 \theta_{2} \cos 2 \theta_{1} & -\sin 2 \theta_{1} \sin 2 \theta_{2}
\end{array}\right)
$$

where $\theta_{1}$ and $\theta_{2}$ represent the rotating angles of the two quarterwave plates $R 1$ and $R 2$, respectively. The detected light intensity can be expressed in a periodic form based on the Fourier analysis as shown below:

$f(x)=a_{0}+\sum_{n=1}^{\infty}\left(a_{n} \cos n \theta_{1}+b_{n} \sin n \theta_{1}\right)$,

where the fundamental frequency $a_{n}$ and $b_{n}(n=1,2 \ldots 12)$ are Fourier amplitudes.

By simultaneously solving Eqs. (2) and (4), we know that the Fourier amplitudes provide linear algebraic equations of the 16 unknown Mueller-matrix elements $m_{i j}$. Twenty-five Fourier amplitudes can be calculated if 30 measurements are taken. Five of the twenty five values are opposite to another five, and four values are always zero. Thus, we can derive the Mueller matrix based on the 16 independent amplitude values.

\subsection{Selection Criteria of the Transmission Microscope}

Although Mueller matrix measurements can be realized by adding polarization optical elements to an ordinary transmission microscope, we need to choose microscopes suitable for the modification. According to our experience, we should choose the transmission microscope with the following three characteristics. First, the microscope should have an external light source that is easily replaced by a multiwavelength collimated light source (CLS) module. Second, the microscope should be an infinity optical system with infinity plan achromatic objectives, making it easier to add the PSA module in the detecting optical path. Finally, there should be a long moving-range sample stage that has spare space for adding the PSG module in the microscope. In this work, we chose a low cost commercial transmitted-light microscope (L2050, LISS Optical Co. Ltd., China). Based on the modulus design, the polarized light microscope can be easily disassembled and upgraded.

\subsection{Selection Criteria of the Light Source}

Monochrome LEDs are promising light sources for the microscopy measurements. They are small, lightweight, low cost, and convenient for working at low voltages. ${ }^{23,24}$ There are six different monochrome LEDs installed in the microscope; the center wavelengths of the LEDs are $455 \mathrm{~nm}$ (royal blue), $475 \mathrm{~nm}$ (blue), $490 \mathrm{~nm}$ (cyan), $535 \mathrm{~nm}$ (green), $590 \mathrm{~nm}$ (amber), and $630 \mathrm{~nm}$ (red), respectively, and each of their full-width at half-maximum is close to $15 \mathrm{~nm}$. 


\subsection{Selection Criteria of the Polarization Modules}

There are many types of instrumental setups used for time sequential polarization modulation, including rotating polarized optical components, ${ }^{25,26}$ photoelastic modulators, ${ }^{27}$ and liquid crystal variable retarder. ${ }^{28}$ The photoelastic modulator is expensive, and the CCD detector that we used cannot take advantage of its high modulation speed. The liquid crystal variable retarder is sensitive to the ambient temperature. Thus, in our system, polarization-state modulations in both PSG and PSA are realized by rotating the wave plates around the optical axis. In the microscope, all the polarized components $P 1, R 1, R 2$, and $P 2$ are placed horizontally. The transmission axes of the polarizers and the fast axes of the quarter-wave plates are parallel to each other; then, the PSG and PSA are modulated by synchronously rotating two quarter-wave retarders at fixed angles per step.

\section{Experimental Setup}

\subsection{General Design of Hardware}

Figure 2(a) shows a general schematic of the modulus design polarization microscope for transmission Mueller matrix imaging. The polarization microscope consists of several major modules: (i) CLS module, (ii) PSG module, (iii) PSA module, and (iv) image receiving module. In the CLS module, the diverging light from a monochrome LED (one of the six LEDs of different colors) is collimated by a set of fixed upright standing convex lenses (GCL-010158A, GCL-010111A, Daheng New Epoch Technology, Inc., China) and turned to the upright direction by a steering mirror $\mathrm{M}$. The parallel beam passes through the PSG module, which consists of a fixed polarizer $P 1$ (LPVISE100-1, Thorlabs, Inc.) and an achromatic quarter-wave plate $R 1$ (WPA4225, 450-650 nm, Union Optic Co., Ltd., China) mounted on a computer controlled motorized rotational stage (PRM1Z8E, Thorlabs, Inc.). The parallel beam with well controlled SoP illuminates the sample $\mathrm{S}$ on the translation stage.

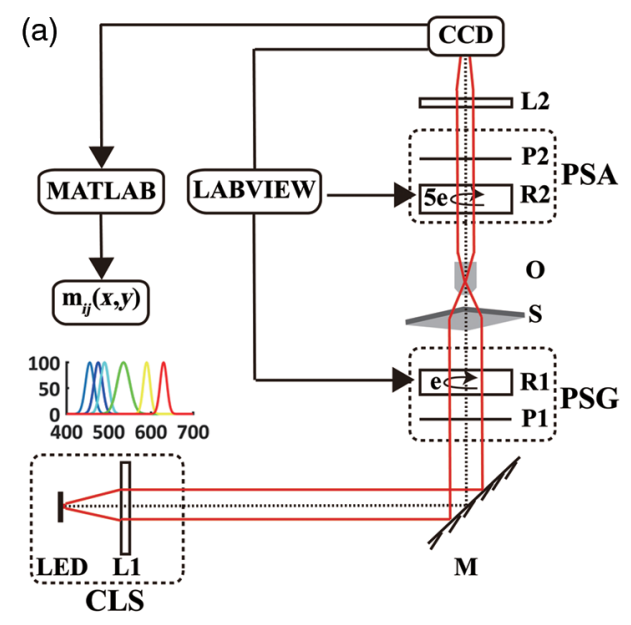

(b)

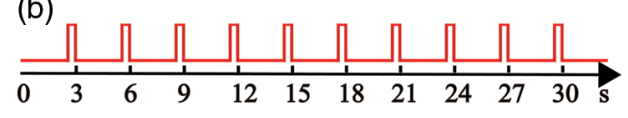

Fig. 2 (a) The schematic diagram of polarization microscope. CLS, monochromatic light source; $P 1$ and $P 2$, polarizers; $R 1$ and $R 2$, quarter-wave plates; $L 1$ and L2, lenses; O, $4 \times, 10 \times, 20 \times, 40 \times$ objectives. (b) The time for image acquisition. The protuberant red lines represent the exposure time range from 0.1 to $1 \mathrm{~ms}$; the following red horizontal lines represent the rotation time of $R 1$ and $R 2$, which is about $3 \mathrm{~s}$.
The transmitted light from the sample is collected by the objective lens $\mathrm{O}$ and then passes though the PSA module, which also consists of an achromatic quarter-wave plate R2 (WPA4225, 450-650 nm, Union Optic, Inc., China) mounted on a computer controlled motorized stage (PRM1Z8E, Thorlabs, Inc.) and a fixed linear polarizer P2 (LPVISE100-1, Thorlabs, Inc.). Finally, the analyzed light is focused and detected by a 12-bit CCD detector (74-0107A, Dynisco, Inc., China). The exposure time takes from 0.1 to $1 \mathrm{~ms}$ for imaging different samples, but the time for setting the PSG and PSA in position takes up to $3 \mathrm{~s}$ as it is limited by the speed of the rotational stage in PSA, as shown in Fig. 2(b). For each measurement, the system takes 30 polarization component images corresponding to different combinations of the PSG and PSA, and then evaluates the 16 Mueller matrix elements following Eqs. (2) and (4). It takes about one and a half minutes for a Mueller matrix measurement.

\subsection{Software for System Control, Data Acquisition and Analysis}

The software system of the polarized microscope is capable of hardware control, data acquisition, data processing, and data analysis. A graphical user interface program for instrument control and data acquisition has been developed using the National Instruments' Labview (Laboratory Virtual Instrument Engineering Workbench) graphical programming platform. We adopt Labview program to control the two high-precision motorized rotation stages and the CCD, as shown in Fig. 1. For each measurement, first, the system control program makes the rotation stages turn to a specified angle separately, making the transmission axes of the polarizers and the fast axes of the quarter-wave plates parallel. Second, the program repeats the image collecting process 30 times, during which the two rotation stages $R 1$ and $R 2$ rotate at preset angles, respectively. Then, the $C C D$ takes one or several repeated images with suitable exposure time ranging from 0.1 to $1 \mathrm{~ms}$. Finally, the Labview program takes the two rotation stages back to their initial positions ready for the next measurement. In this Labview program, we can set the number for repetitive data collections, rotation angles for each step, exposure time, and data storage location. With a computer controlling the program, the measurement time is mainly limited by the rotating speeds of the motors. The current microscope takes a minimum of $91 \mathrm{~s}$ to complete a Mueller matrix measurement, $90 \mathrm{~s}$ for the rotations of the wave plates, and about $1 \mathrm{~s}$ for calculating the Mueller matrix from the 30 polarization component images. We use MATLAB for both data processing and for calibrations that will be discussed later.

\section{System Optimization and Calibration}

\subsection{Optimization of Imaging Receiving Module}

The calculation of the Mueller matrix is based on the light intensity value from the CCD. To ensure accurate light intensity detection and avoid overexposure, the illumination intensity is controlled to make the maximum pixel values of the polarization component images in CCD below 4000. The error of light intensity value is mainly related to the stability of light source and the noise of optoelectronic detectors. In this section, we take the light intensity value of a single measurement and the average value of $2,4,6,8,10,12,14$, and 16 repeated measurement results as experiment results. Then, we calculate the mean value and the variance of 10 parallel experiments to estimate the stability of the measured light intensity values. Using 630-nm (red) 
LED as illumination light, we display the values and the variance of pixels in the middle of the image with the form of errorbar in Fig. 3. There is a significant decrease in the variance of the direct intensity values and the average values of $2,4,6,8$, and 10 repeated images, as shown in Figs. 3(a)-3(f). In addition, Figs. 3(f)-3(i) indicate that the average value of over 10 repeated measurements results in a very small improvement to the stability of light intensity value. We get the same results with other wavelengths of illumination light. Thus, we use the average value of 10 repeated results to substitute the single measurement result in the Mueller matrix measurements.

\subsection{Modeling Method of Polarization Calibration}

Calibrations of the polarization optics are very important for accurate Mueller matrix measurements. The polarization elements $R 1, R 2$, and $P 2$ have errors associated with their initial azimuthal alignment with respect to the transmission axis of $P 1$. In addition, the retarders may slightly deviate from the retardance of $90 \mathrm{deg}$. We build a simple model based on the study of Goldstein, ${ }^{29}$ which include five potential error sources, respectively, represented by $\varepsilon_{1}, \varepsilon_{2}, \varepsilon_{3}, \delta_{1}$, and $\delta_{2}$, as shown in Fig. 1 , and can be expressed as shown below:

$\mathbf{S}_{\text {out }}=\mathbf{M}_{P 2}\left(\varepsilon_{5}\right) \mathbf{M}_{R 2}\left(\delta_{2}, \varepsilon_{4}\right) \mathbf{M}_{\text {sample }} \mathbf{M}_{R 1}\left(\delta_{1}, \varepsilon_{3}\right) \mathbf{M}_{P 1} \mathbf{S}_{\mathrm{in}}$.

The Mueller matrix $M_{R 1}$ and $M_{P 2}$ containing the errors can be expressed as shown below:

$$
M_{P 2}=\frac{1}{2} R\left(\varepsilon_{3}\right) M_{P 1} R\left(-\varepsilon_{3}\right),
$$

$M_{R 1}=\left(\begin{array}{cccc}1 & 0 & 0 & 0 \\ 0 & \cos ^{2} 2\left(\theta_{2}+\varepsilon_{2}\right)+\sin ^{2} 2\left(\theta_{2}+\varepsilon_{2}\right) \cos \left(\delta_{1}\right) & \cos 2\left(\theta_{2}+\varepsilon_{2}\right) \sin 2\left(\theta_{2}+\varepsilon_{2}\right)\left[1-\cos \left(\delta_{1}\right)\right]-\sin 2\left(\theta_{2}+\varepsilon_{2}\right) \sin \left(\delta_{1}\right) \\ 0 & \cos 2\left(\theta_{2}+\varepsilon_{2}\right) \sin 2\left[\theta_{2}+\varepsilon_{2}\right)\left(1-\cos \left(\delta_{1}\right)\right] & \sin ^{2} 2\left(\theta_{2}+\varepsilon_{2}\right)+\cos ^{2} 2\left(\theta_{2}+\varepsilon_{2}\right) \cos \left(\delta_{1}\right) & \cos 2\left(\theta_{2}+\varepsilon_{2}\right) \sin \left(\delta_{1}\right) \\ 0 & \sin 2\left(\theta_{2}+\varepsilon_{2}\right) \sin \left(\delta_{1}\right) & -\cos 2\left(\theta_{2}+\varepsilon_{2}\right) \sin \left(\delta_{1}\right) & \cos \left(\delta_{1}\right)\end{array}\right)$

and $M_{R 2}$ can be expressed by analogizing $M_{R 1}$. By contrasting Eqs. (2) and (5), we can derive the 16 unknown Mueller-matrix elements $m_{i j}$ with Fourier amplitudes and errors. When air is used as the reference sample, the Mueller matrix should be strictly diagonal. Through inverse operations, the five errors can be calculated from the Fourier coefficient in the following equations:
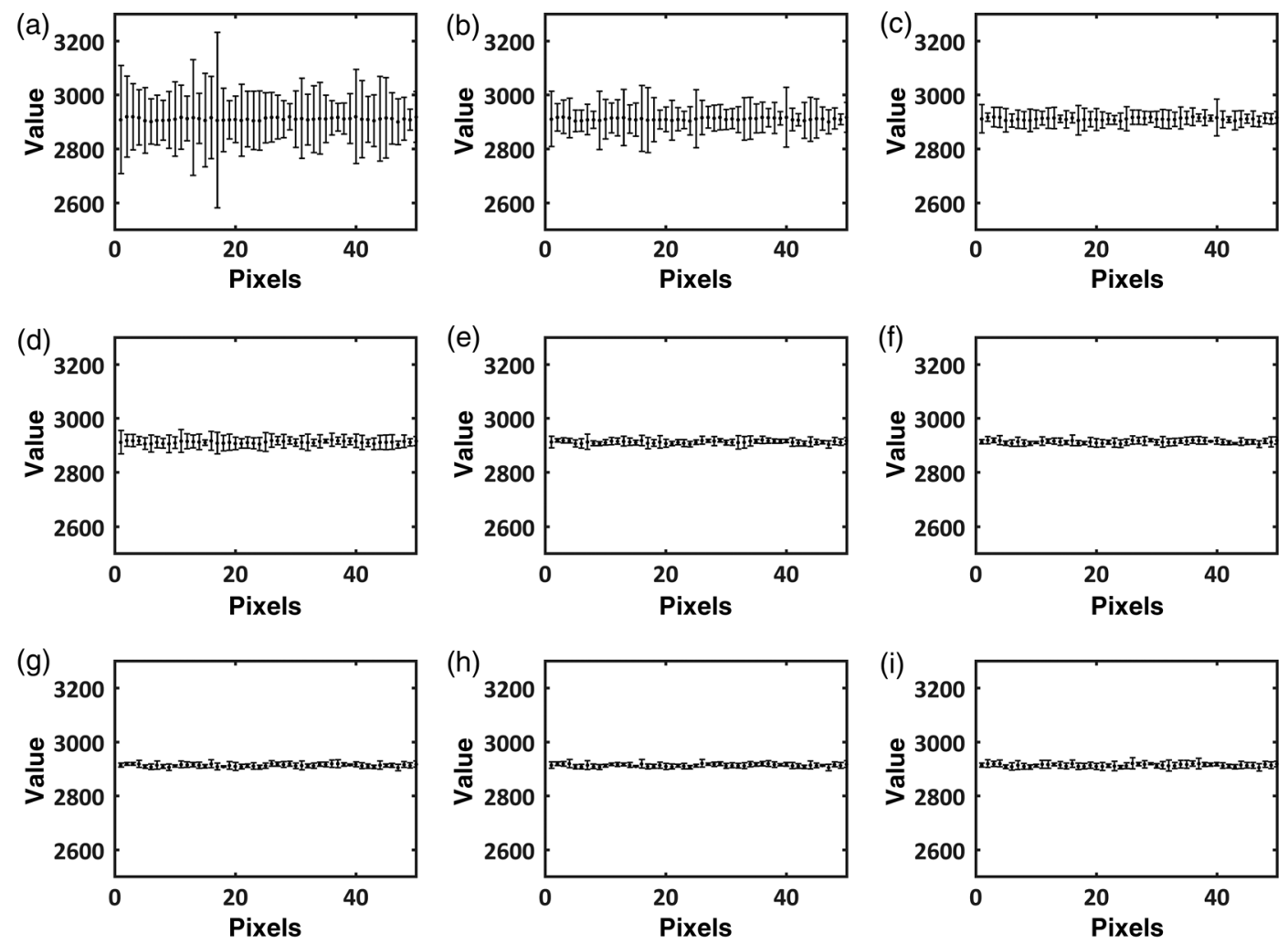

Fig. 3 (a) The error bar of the light intensity value of a single measurement based on 10 parallel experiments. (b)-(i) The error bar of the average value of $2,4,6,8,10,12,14$, and 16 repeated measurement results based on 10 parallel experiments. 


$$
\begin{aligned}
\delta_{1}= & \cos ^{-1}\left(\frac{a_{10} \cos \alpha_{9}-a_{8} \cos \alpha_{11}}{a_{10} \cos \alpha_{9}+a_{8} \cos \alpha_{11}}\right), \\
\delta_{2}= & \cos ^{-1}\left[\frac{a_{2} \cos \alpha_{9}-a_{8} \cos \left(4 \varepsilon_{3}-2 \varepsilon_{5}\right)}{a_{2} \cos \alpha_{9}+a_{8} \cos \left(4 \varepsilon_{3}-2 \varepsilon_{5}\right)}\right], \\
\varepsilon_{3}= & \frac{1}{4} \tan ^{-1}\left(\frac{b_{8}}{a_{8}}\right)-\frac{1}{4} \tan ^{-1}\left(\frac{b_{10}}{a_{10}}\right), \\
\varepsilon_{4}= & \frac{1}{2} \tan ^{-1}\left(\frac{b_{2}}{a_{2}}\right)-\frac{1}{2} \tan ^{-1}\left(\frac{b_{6}}{a_{6}}\right)+\frac{1}{4} \tan ^{-1}\left(\frac{b_{8}}{a_{8}}\right) \\
& -\frac{1}{4} \tan ^{-1}\left(\frac{b_{10}}{a_{10}}\right), \\
\varepsilon_{5}= & \frac{1}{2} \tan ^{-1}\left(\frac{b_{2}}{a_{2}}\right)+\frac{1}{2} \tan ^{-1}\left(\frac{b_{8}}{a_{8}}\right)-\frac{1}{2} \tan ^{-1}\left(\frac{b_{10}}{a_{10}}\right) .
\end{aligned}
$$

By taking many measurements, we can reduce $\varepsilon_{1}, \varepsilon_{2}$, and $\varepsilon_{3}$ to $1 \mathrm{deg}$, and the maximum errors of the Mueller matrix elements with angular calibration can be less than 0.01 .

\subsection{Eigenvalue Calibration Method}

After the modeling method calibration, the errors associated with the polarized optics in the Mueller matrix measurements can be mostly eliminated. It is noteworthy that the objective lens also affects Mueller matrix measurements. Although objective lenses are usually regarded as nonpolarization optics, sometimes their polarization properties show up as birefringence and depolarization. The birefringence of glass lens is usually small but could be big enough to affect the accuracy of Mueller matrix measurements if miniaturized or organic material lenses are used. Wolfe and Chipman ${ }^{30}$ found the annealing of lens generated by depolarization phenomenon and Wood and Elson ${ }^{31}$ found that the optical quartz window had an impact on polarization imaging. We use the Mueller matrix to characterize the polarization property of an objective. Five standard samples, i.e., air, polarizer in $45 \mathrm{deg}$, polarizer in $90 \mathrm{deg}$, quarter-wave plate in $45 \mathrm{deg}$, and quarter-wave plate in $90 \mathrm{deg}$, are used to evaluate the Mueller matrix of an objective using the eigenvalue calibration method..$^{32,33}$ The Mueller matrix of air is a unit matrix, and the measured results of air and other samples can be expressed as shown below:

$M_{0}=T_{\text {air }} X$,

$M_{i}=T_{i} X$

where $M_{0}$ represents the experimental Mueller matrix of the air and $M_{i}$ represents the experimental Mueller matrix of the $i^{\prime}$ th type of standard samples. $T_{\text {air }}$ represents the Mueller matrix of the air and $T_{i}$ represents the Mueller matrix of the $i^{\prime}$ th standard sample. $X$ represents the unknown Mueller matrix of the objective. We used experimental result $M_{0}$ and $M_{i}$ to define a new matrix $D_{i}=M_{0}^{-1} M_{i}=X^{-1} T_{i} \mathrm{X}$ and obtain the following equation:

$\mathrm{X} D_{i}=T_{i} X$

by multiplying the inverse of matrix $X$ on both sides of the equation. Equation (11) formed a linear system whose optimal solution can be derived by the least square method. We define
Table 1 The calculated optimal Mueller matrix of the $4 \times$ objective.

\begin{tabular}{lccc}
\multicolumn{4}{c}{ Mueller matrix after angular calibration } \\
\hline 1.000 & -0.002 & 0.002 & 0.002 \\
0.000 & 0.989 & -0.004 & 0.002 \\
0.000 & 0.000 & 0.991 & -0.023 \\
0.000 & -0.004 & 0.017 & 0.982 \\
\hline
\end{tabular}

the linear operator $P_{i}(X)=T_{i} X-X D_{i}$ to describe the different value.

For convenience of calculation, $P_{i}$ and $X$ are arranged in rows and projected into vectors, which are shown as $\left[P_{i}(X)\right]^{(16)}$ and $X^{(16)}$ separately in the following equation:

$\left[P_{i}(X)\right]^{(16)}=Y_{i}^{(16)}=H_{i}^{(16,16)} X^{(16)}$

and the coefficient matrix $H_{i}^{(16,16)}$, whose size is $16 \times 16$, is calculated. For convenience, we use $Y_{i}^{(16)}$ to represent $\left[P_{i}(X)\right]^{(16)}$, as shown in Eq. (12). The square sum of the difference values can be expressed as shown below:

$\left[\mathrm{Y}_{i}^{(16)}\right]^{2}=\left[X^{(16)}\right]^{T}\left[\mathrm{H}_{i}^{(16,16)}\right]^{T} \mathrm{H}_{i}^{(16,16)} X^{(16)}=\left[X^{(16)}\right]^{T} K_{i} X^{(16)}$,

and vector $X^{(16)}$ is the eigenvector of the real symmetric positive semidefinite matrix $K_{i}$.

We define $K_{\text {tot }}=\sum_{i} K_{i}$ to represent the sum of $K_{i}$ and find the smallest eigenvalue and the corresponding eigenvector, which can be rearranged into the Mueller matrix of the objective, as the calculated result of the $4 \times$ objective shown in Table 1.

\subsection{Optimization of Rotation Angles}

After the calibration as above, the accuracy of the Mueller matrix measurement is enhanced, but errors still exist in the system. Fortunately, a good measurement system can reduce the effects of errors to the results, and the condition number $(\mathrm{CN})$ of the measurement matrix is a metric of the stability of the system. We calculate the $\mathrm{CN}$ of the Mueller matrix microscope with the Mueller matrix of the objective when the rotation angles of the two quarter-wave plates change, as shown in Fig. 4. For the smallest possible $\mathrm{CN}$ and the convenience of operation, we chose the smallest $\theta_{1}$ and $\theta_{2}$, so $\theta_{1}=6 \mathrm{deg}, \theta_{2}=30 \mathrm{deg}$, and $\mathrm{CN}=2.573$.

\subsection{Solution to Beam Drifting}

We find that the images received by the detector cannot line up perfectly. The cause of this is that there are two rotation polarization devices in the system, and the surface of the polarization components may be unparallel. The beam of light drifts, and the measurement regions form dislocations because of rotation, as shown in Fig. 5. We calculate in Eq. (14) the relationship between the offset of the image $L$ and the angle $\gamma$ between the optical path and the central axis of the rotating optical component in PSA. We enhance the accuracy of image matching 


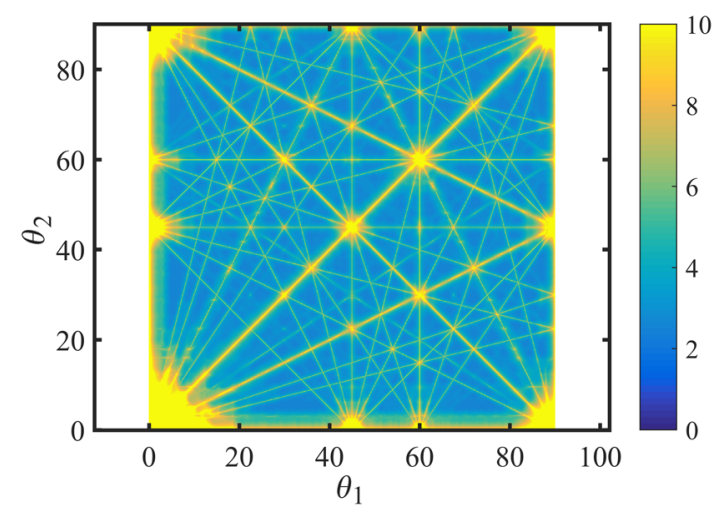

Fig. 4 The image of the $\mathrm{CN}$ of the Mueller matrix microscope when the rotation angles of the two quarter-wave plates change. The horizontal axis shows the rotation angle of $\theta 1$ in degree, and the vertical axis shows the rotation angle of $\theta 2$ in degree.

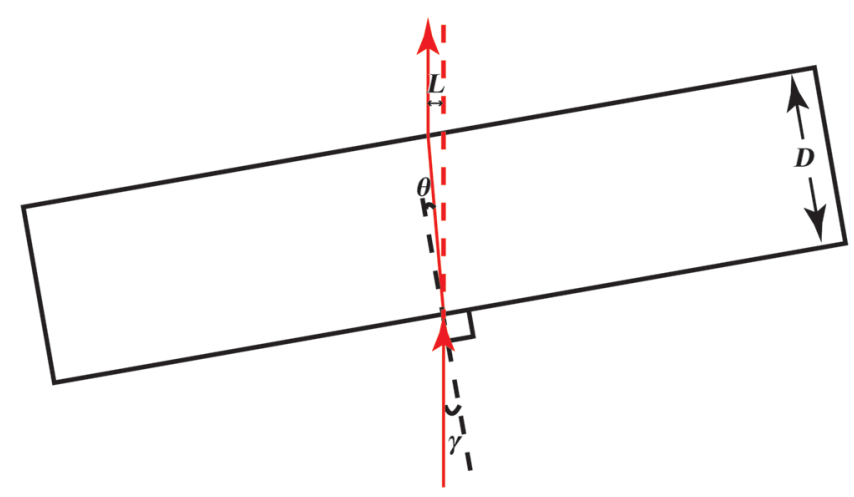

Fig.5 The illustration of light drift. When polarization components are unparallel, the beam of light drifts and the measurement regions form dislocations. $D$, the thickness of the wave plate; $L$, the distance of deviation; $\gamma$, the angle between the optical path and the central axis of the rotating optical component in PSA; and $\theta$, the angle of refraction. before calculation and show the original image and the improved image of MMD parameter $D$ of porous alumina ${ }^{34}$ in Fig. 6. Comparing the results shown in Figs. 6(a) and 6(b), the MMD parameter $D$ of porous alumina with image registration has less noise and a clearer boundary, which demonstrates that our strategy can reduce part of the effects due to light beam drift:

$$
L=\frac{D * \sin (\gamma-\theta)}{\cos \gamma} .
$$

\section{System Test Result and Experiment Result}

\subsection{System Test Result}

In addition to air, we also measured a polarizer as a standard sample. Table 2 shows the measurement results of the polarizer for six different wavelengths, which demonstrate that the error for each wavelength is below 0.01 after calibration. Therefore, the measurement results of our polarized light microscope are reliable.

\subsection{Experimental Result}

In this study, we use histopathologic slides as samples. The histopathologic samples used in this study are nonstained, dewaxing sections of human liver cancer tissues, which were prepared and provided by Shenzhen Sixth People's (Nanshan) Hospital. We display the images of the Mueller matrix polarization decomposition (MMPD) ${ }^{35}$ retardance $\delta$ of human liver tissue with different wavelengths in Fig. 7. During the development process of liver cancerous tissues, the accompanied inflammatory reactions can result in fibrosis and cirrhosis, which can be reflected by the values of the MMPD parameter retardance $\delta^{2}$. Therefore, compared with the normal regions, the abnormal regions of the liver tissue slides contain much more fibrous structures, resulting in larger MMPD parameter retardance $\delta$ values. The normal regions in liver tissue are mainly the blue regions with low retardance, while the abnormal regions are

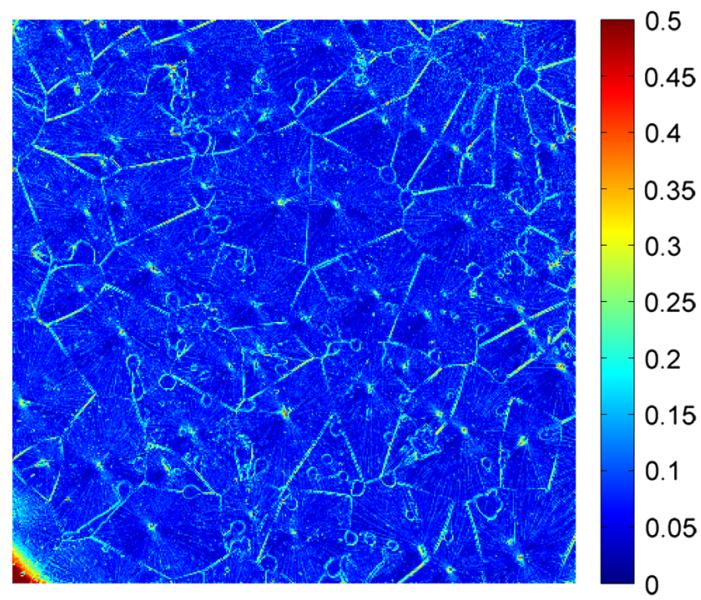

(a)

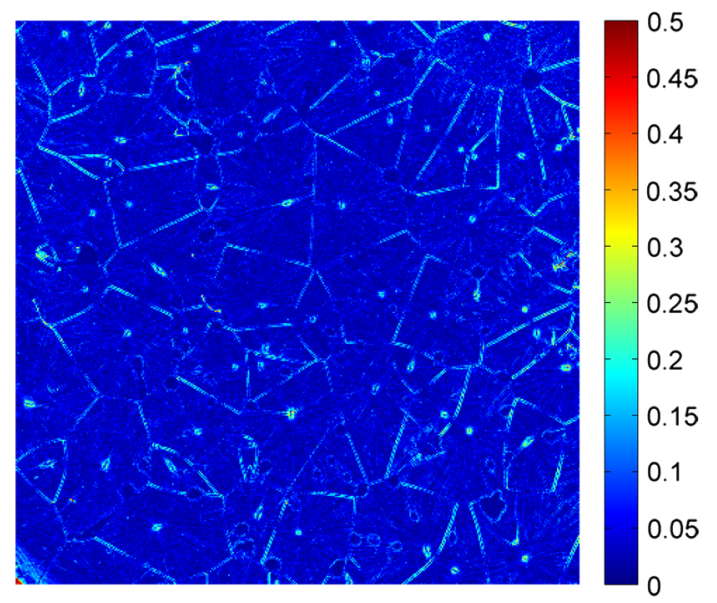

(b)

Fig. 6 (a) The original result of porous alumina and (b) the result of porous alumina with image registration. 
Table 2 (a)-(f) The final Mueller matrix of polarizer after calibration with light in $455 \mathrm{~nm}$ (royal blue), $475 \mathrm{~nm}$ (blue), $490 \mathrm{~nm}$ (cyan), $535 \mathrm{~nm}$ (green), $590 \mathrm{~nm}$ (amber), and $630 \mathrm{~nm}$ (red) respectively.

\begin{tabular}{|c|c|c|c|c|c|c|c|c|c|c|c|c|c|c|}
\hline \multirow[t]{4}{*}{ (a) } & 1.000 & 1.007 & -0.002 & 0.004 & (b) & 1.000 & 1.000 & 0.000 & 0.002 & (c) & 1.000 & 1.004 & 0.006 & 0.003 \\
\hline & 1.003 & 0.994 & -0.007 & 0.000 & & 0.995 & 1.007 & -0.008 & -0.001 & & 0.993 & 0.999 & 0.001 & -0.001 \\
\hline & -0.005 & 0.004 & -0.007 & 0.001 & & -0.002 & 0.008 & -0.003 & -0.001 & & 0.002 & 0.003 & 0.004 & 0.009 \\
\hline & -0.002 & -0.009 & -0.005 & -0.002 & & -0.000 & -0.009 & -0.007 & 0.001 & & -0.001 & -0.008 & -0.009 & 0.000 \\
\hline \multirow[t]{4}{*}{ (d) } & 1.000 & 0.993 & -0.002 & 0.002 & (e) & 1.000 & 0.997 & 0.001 & 0.000 & (f) & 1.000 & 0.998 & -0.002 & 0.003 \\
\hline & 0.993 & 1.003 & 0.006 & 0.001 & & 0.995 & 1.000 & 0.005 & 0.007 & & 0.999 & 0.996 & -0.001 & -0.004 \\
\hline & 0.003 & -0.008 & -0.006 & 0.003 & & 0.000 & 0.009 & 0.004 & 0.008 & & 0.000 & -0.009 & 0.007 & 0.003 \\
\hline & -0.003 & -0.004 & -0.008 & 0.000 & & -0.004 & 0.000 & -0.003 & 0.002 & & -0.002 & -0.004 & -0.006 & 0.000 \\
\hline
\end{tabular}

the red regions with high retardance and marked with black lines in Fig. 7(a). For quantitative analysis, we calculate the mean retardance value of the normal regions and abnormal regions. When the wavelength of light decreases from 630 to $455 \mathrm{~nm}$, retardance $\delta$ of normal regions increases from 0.18 to 0.28 , retardance $\delta$ of abnormal regions increases from 0.54 to 0.81 , and the retardance difference between the abnormal and normal regions increases from 0.36 to 0.53 correspondingly, as shown in Fig. 8. We can use retardance $\delta$ as a feature parameter to distinguish abnormal regions from normal regions. In this experiment, the retardance difference of $455 \mathrm{~nm}$ light is $47 \%$ higher than that of $630-\mathrm{nm}$ light, which means that $455-\mathrm{nm}$ light is a better choice because the shorter wavelength is more sensitive to the change of retardance $\delta$. Because the scattering and absorption characteristics of different samples are not the same, longer wavelength light may have advantages in some other cases. For different samples and different feature parameters, we need to choose a suitable wavelength to measure the Mueller matrix to achieve the best effect. Fortunately, a multiwavelength polarization microscope can give us multiple choices and provide much more useful information compared with a monochromatic Mueller matrix microscope. This work was approved by the Ethics Committee of the Shenzhen Sixth People's (Nanshan) Hospital. (a)

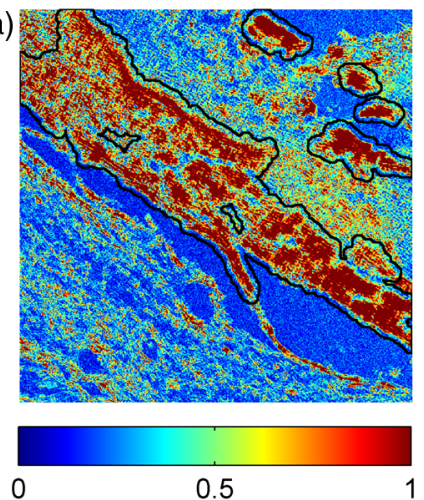

(d)

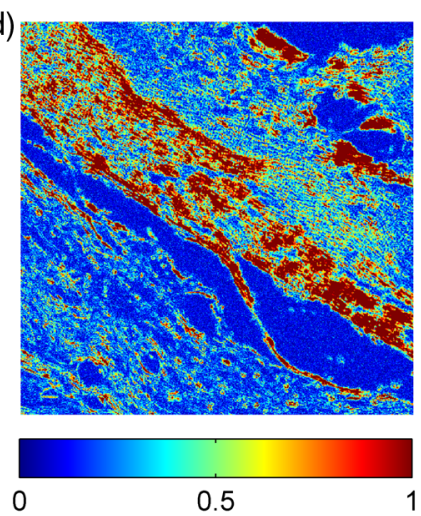

(b)

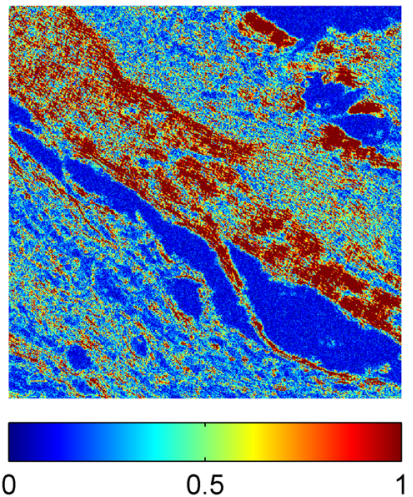

(e)

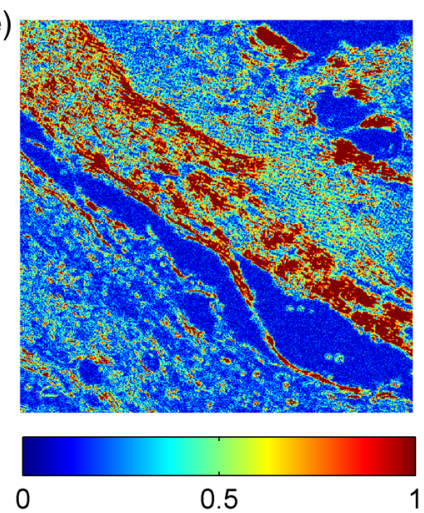

(c)

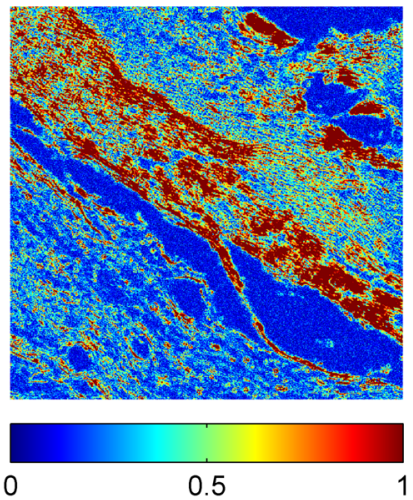

(f)

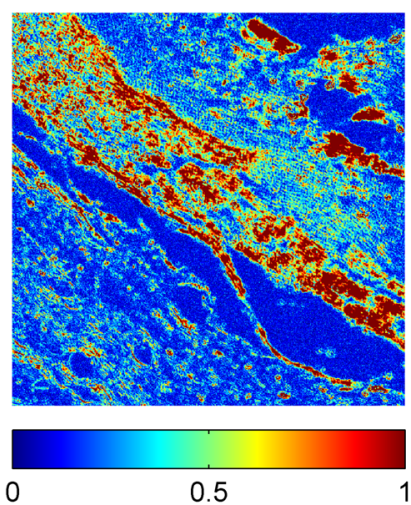

Fig 7 (a)-(f) The images of the MMPD parameter $\delta$ of human liver tissue slices with light in $455 \mathrm{~nm}$ (royal blue), $475 \mathrm{~nm}$ (blue), $490 \mathrm{~nm}$ (cyan), $535 \mathrm{~nm}$ (green), $590 \mathrm{~nm}$ (amber), and $630 \mathrm{~nm}$ (red), respectively; the abnormal regions in these images are indicated by black lines in (a). 


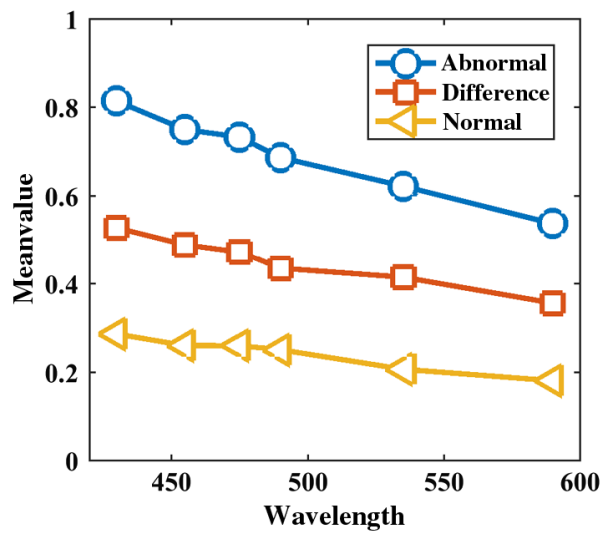

Fig 8 The mean values and the difference values of the MMPD parameter $\delta$ of human liver tissue slices in abnormal regions and normal regions when the wavelength of light changes from 430 to $590 \mathrm{~nm}$; the mean values of abnormal regions are in blue line and circular marker; the mean values of normal regions are in yellow line and triangle marker; the difference values between the abnormal regions and normal regions are in red line and square marker.

\section{Conclusions}

In conclusion, we have demonstrated that an ordinary optical microscope can be transformed to a multiwavelength polarization microscope for Mueller matrix measurements just by replacing the light source and adding a set of PSG and PSA. The maximum errors for the absolute values of Mueller matrix elements can be reduced to about 0.01 after calibration. By measuring air, polarizer, and histopathologic slides, we verify the reliability of the polarized light microscope and find that we can obtain rich information on the microstructure and optical properties of samples. The Mueller matrix microscope is a very versatile instrument and will have a wide range of applications.

\section{Disclosures}

The authors have no relevant financial interests in this article and no potential conflicts of interest to disclose.

\section{Acknowledgments}

This research was supported by the National Natural Science Foundation of China (NSFC) under Grant Nos. 11374179, 61527826, and 61405102 and Science and Technology Project of Shenzhen under Grant Nos. JCYJ20170412170814624 and GJHZ20150316160614844.

\section{References}

1. R. A. Chipman, "Handbook of optics," in Polarimetry, 2nd ed., M. Bass, Ed., p. 2, McGraw Hill, New York (1995).

2. Y. Wang et al., "Mueller matrix microscope: a quantitative tool to facilitate detections and fibrosis scorings of liver cirrhosis and cancer tissues," J. Biomed. Opt. 21(7), 071112 (2016).

3. G. C. Giakos et al., "Electro-optical polarimetric imaging system for characterization of resident space objects," J. Spacecr. Rockets 52(4), 1112-1128 (2015).

4. B. Peng, T. Ding, and P. Wang, "Propagation of polarized light through textile material," Appl. Opt. 51(26), 6325-6334 (2012).

5. H. Wang et al., "Polarization sensitive optical coherence microscopy for brain imaging," Opt. Lett. 41(10), 2213-2216 (2016).

6. S. F. Pellicori and E. Burke, "Passive multispectral imaging polarimeter for remote atmospheric and surface studies: design based on optical coatings," Appl. Opt. 55(6), 1291-1301 (2016).

7. F. Goudail and M. Boffety, "Optimal configuration of static polarization imagers for target detection," J. Opt. Soc. Am. A 33(1), 9-16 (2016).
8. R. Oldenbourg, "A new view on polarization microscopy," Nature 381(6585), 811-812 (1996).

9. S. Ross et al., "Quantitative image analysis of birefringent biological material," J. Microsc. 187(1), 62-67 (1997).

10. R. Weaver, "Rediscovering polarized light microscopy," Am. Lab. 35(20), 55-61 (2003).

11. R. Oldenbourg, "Polarized light field microscopy: an analytical method using a microlens array to simultaneously capture both conoscopic and orthoscopic views of birefringent objects," J. Microsc. 231(3), 419-432 (2008).

12. M. Antonelli et al., "Mueller matrix imaging of human colon tissue for cancer diagnostics: how Monte Carlo modeling can help in the interpretation of experimental data," Opt. Express 18(10), 10200-10208 (2010).

13. A. Pierangelo et al., "Polarimetric imaging of uterine cervix: a case study," Opt. Express 21(12), 14120-14130 (2013).

14. N. Ghosh et al., "Mueller matrix decomposition for polarized light assessment of biological tissues," J. Biophotonics 2(3), 145-156 (2009).

15. M. Dubreuil et al., "Mueller matrix polarimetry for improved liver fibrosis diagnosis," Opt. Lett. 37(6), 1061-1063 (2012).

16. Y. Wang et al., "Differentiating characteristic microstructural features of cancerous tissues using Mueller matrix microscope," Micron 79, 8-15 (2015).

17. D. Dixit et al., "Optical critical dimension metrology for directed selfassembly assisted contact hole shrink," J. Micro/Nanolith. MEMS MOEMS 15(1), 014004 (2016).

18. Y. Dong et al., "Quantitatively characterizing the microstructural features of breast ductal carcinoma tissues in different progression stages by Mueller matrix microscope," Biomed. Opt. Express 8(8), 3643-3655 (2017).

19. R. Azzam, "Photopolarimetric measurement of the Mueller matrix by Fourier analysis of a single detected signal," Opt. Lett. 2(6), 148-150 (1978).

20. J. Chang et al., "Division of focal plane polarimeter-based $3 \times 4$ Mueller matrix microscope: a potential tool for quick diagnosis of human carcinoma tissues," J. Biomed. Opt. 21(5), 056002 (2016).

21. Y. Liu et al., "Complementary fluorescence-polarization microscopy using division-of-focal-plane polarization imaging sensor," J. Biomed. Opt. 17(11), 116001 (2012).

22. H. He et al., "Two-dimensional and surface backscattering Mueller matrices of anisotropic sphere-cylinder scattering media: a quantitative study of influence from fibrous scatterers," J. Biomed. Opt. 18(4), 046002 (2013).

23. S. Ho et al., "Review of recent progress in multilayer solution-processed organic light-emitting diodes," J. Photonics Energy 5(1), 057611 (2015).

24. G. Li et al., "GaN-based light-emitting diodes on various substrates: a critical review," Rep. Prog. Phys. 79(5), 056501 (2016).

25. E. Bernabeu and J. J. Gil, "An experimental device for the dynamic determination of Mueller matrices," J. Opt. 16(3), 139-141 (1985).

26. K. Ichimoto et al., "Photopolarimetric measurement system of Mueller matrix with dual rotating waveplates," Publ. Natl. Astron. Obs. Jpn. 9, 11-19 (2016).

27. O. Arteaga et al., "Mueller matrix polarimetry with four photoelastic modulators: theory and calibration," Appl. Opt. 51(28), 6805-6817 (2012).

28. A. De Martino et al., "Optimized Mueller polarimeter with liquid crystals," Opt. Lett. 28(8), 616-618 (2003).

29. D. H. Goldstein, "Mueller matrix dual-rotating retarder polarimeter," Appl. Opt. 31(31), 6676-6683 (1992).

30. J. Wolfe and R. A. Chipman, "Reducing symmetric polarization aberrations in a lens by annealing," Opt. Express 12(15), 3443-3451 (2004).

31. T. C. Wood and D. S. Elson, "Polarization response measurement and simulation of rigid endoscopes," Biomed. Opt. Express 1(2), 463-470 (2010).

32. E. Compain, S. Poirier, and B. Drevillon, "General and self-consistent method for the calibration of polarization modulators, polarimeters, and Mueller-matrix ellipsometers," Appl. Opt. 38(16), 3490-3502 (1999).

33. A. D. Martino et al., "General methods for optimized design and calibration of Mueller polarimeters," Thin Solid Films 455, 112-119 (2004).

34. Y. Fan et al., "Optical waveguide sensor based on silica nanotube arrays for label-free biosensing," Biosens. Bioelectron. 67, 230-236 (2015).

35. S. Y. Lu and R. A. Chipman, "Interpretation of Mueller matrices based on polar decomposition," J. Opt. Soc. Am. A 13(5), 1106-1113 (1996).

Biographies for the authors are not available. 